\title{
Optical Transitions in Single-Wall Boron Nitride Nanotubes
}

\author{
J. S. Lauret, ${ }^{*}$ R. Arenal, F. Ducastelle, and A. Loiseau \\ Laboratoire d'Etude des Microstructures, ONERA-CNRS, BP 72, 92322 Châtillon Cedex, France
}

M. Cau, B. Attal-Tretout, and E. Rosencher

Département de Mesures Physiques, ONERA, Chemin de la Hunière, 91761 Palaiseau Cedex, France

L. Goux-Capes

Laboratoire d'Electronique Moléculaire, SCM-DRECAM-DSM, CEA Saclay, 91191 Gif-sur-Yvette Cedex, France

(Received 26 July 2004; published 27 January 2005)

\begin{abstract}
Optical transitions in single-wall boron nitride nanotubes are investigated by means of optical absorption spectroscopy. Three absorption lines are observed. Two of them (at 4.45 and $5.5 \mathrm{eV}$ ) result from the quantification involved by the rolling up of the hexagonal boron nitride $(h-\mathrm{BN})$ sheet. The nature of these lines is discussed, and two interpretations are proposed. A comparison with single-wall carbon nanotubes leads one to interpret these lines as transitions between pairs of van Hove singularities in the one-dimensional density of states of boron nitride single-wall nanotubes. But the confinement energy due to the rolling up of the $h$-BN sheet cannot explain a gap width of the boron nitride nanotubes below the $h$-BN gap. The low energy line is then attributed to the existence of a Frenkel exciton with a binding energy in the $1 \mathrm{eV}$ range.
\end{abstract}

DOI: 10.1103/PhysRevLett.94.037405

PACS numbers: 78.67.Ch, 78.30.Fs, 78.40.Fy

Since the beginning of the 1990s, the interest in nanoobjects has been increasing exponentially. For instance, carbon nanotubes, discovered in 1991 [1], are studied in various domains of physics, such as mechanics [2], electronics [3], optoelectronics [4], and optics [5-10]. A single-wall carbon nanotube (C-SWNT) consists in a rolled up graphene sheet and can be either semiconducting or metallic, depending on the way the graphene sheet is rolled up. The gap of the semiconducting nanotubes is typically about $0.7 \mathrm{eV}$, depending on the nanotube diameter [11]. In 1996, a new kind of nanotube has been synthesized: single-wall boron nitride nanotubes (BN-SWNTs) $[12,13]$. A BN-SWNT consists in a rolled up hexagonal boron nitride sheet. Because bulk hexagonal boron nitride is a wide gap III-V semiconductor $(5.8 \mathrm{eV})$ [14], BNSWNTs are expected to be one-dimensional wide gap semiconductors. Therefore BN-SWNTs are complementary to carbon nanotubes and constitute promising materials for applications in nanoelectronics or optoelectronics (blue-light emitters, excitonic lasers, etc.).

Some experimental studies on boron nitride nanotubes have been reported. For instance, structural investigations by means of high resolution transmission electronic microscopy (HRTEM) [13,15], transport experiments [16], or scanning tunneling spectroscopy $[17,18]$ have been performed. Until now, no experimental measurements of BNSWNTs optical transitions have been reported. However, their knowledge is a key point for the understanding of the fundamental electronic processes in this one-dimensional material. Scanning tunneling spectroscopy and electron energy loss spectroscopy experiments have been performed on (more complex) double-wall and multiwall boron nitride nanotubes [17-19]. Band gaps larger than $4 \mathrm{eV}$ are reported, and the one-dimensional feature of boron nitride nanotubes is confirmed. Some theoretical works on the electronic properties of BN-SWNTs have also been reported [20-22]. Most of these references predict a band gap between 5.5 and $6 \mathrm{eV}$, using the tightbinding method or $a b$ initio calculations.

In this Letter, we report the first measurements of optical transitions in BN-SWNTs. These measurements have been performed on an assembly of BN-SWNTs by means of optical absorption spectroscopy. The optical absorption spectrum exhibits three lines at 4.45, 5.5, and $6.15 \mathrm{eV}$. The two first lines are not observed in the hexagonal boron nitride $(h-\mathrm{BN})$ sheet. They are attributed to the optical transitions of BN-SWNTs. Two interpretations of the nature of these two lines are discussed below. As for singlewall carbon nanotubes, these lines can be interpreted as transitions between pairs of van Hove singularities in the one-dimensional density of states. An interpretation of the $4.45 \mathrm{eV}$ line in terms of an excitonic transition will be preferred. The binding energy of this Frenkel exciton is in the $1 \mathrm{eV}$ range.

BN-SWNTs are obtained by the laser vaporization of a catalyst-free boron nitride target under a nitrogen atmosphere [13]. A CW- $\mathrm{CO}_{2}$ laser $(10.6 \mu \mathrm{m}, 1 \mathrm{~kW})$ is focused on a rotating $\mathrm{BN}$ target and $\mathrm{BN}-\mathrm{SWNTs}$ grow continuously. The raw material is investigated by means of HRTEM (see Fig. 1), electron energy loss spectroscopy [23], Raman spectroscopy [24], and x-ray diffraction [13]. The raw material is made up of single-wall boron nitride nanotubes, hexagonal boron nitride particles, boron particles encapsulated in a shell of a few $h$-BN layers, and boric 


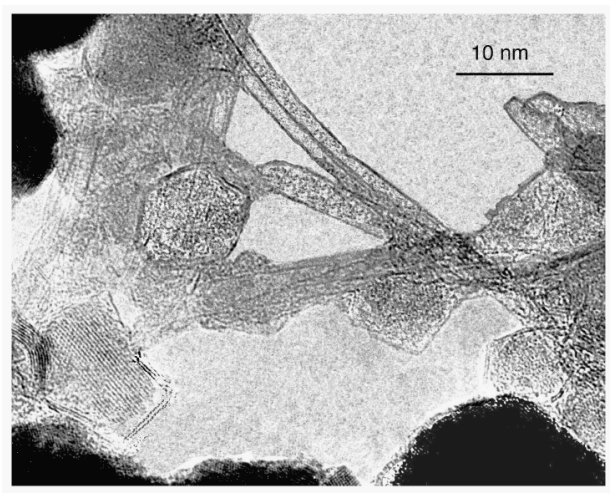

FIG. 1. HRTEM image of the raw material of boron nitride nanotubes. Single-wall boron nitride nanotubes are observed. $h$-BN particles and boron particles encapsulated in a shell of a few $h$-BN layers are also observed.

acid (50\% in weight). The average diameter of the nanotubes is estimated at $1.4 \mathrm{~nm}$, measured by transmission electronic microscopy. In order to enhance the nanotube density in the sample, the raw material has been purified. The purification consists in a filtration step following by a drying step. A suspension of the raw material in 1,2dichlorobenzene or in ethanol, in which boric acid is soluble, is filtered with a $0.2 \mu \mathrm{m}$ pore polytetrafluoroethylene filter. The most dominant signature of boric acid in Raman spectroscopy is a peak centered at $880 \mathrm{~cm}^{-1}$ [24]. After the filtration step no evidence of this signature can be observed, suggesting that the majority of boric acid has been removed. The filter is then dried at $60^{\circ} \mathrm{C}$ during one night in order to remove any trace of the solvent. The purified material has a pronounced filamentous aspect suggesting that the BN-SWNT concentration has increased thanks to the purification step. The purified material is then dissolved in ethanol, and the suspension is sprayed on a quartz substrate which is transparent in the UV-Vis range. Optical absorption spectra are performed using a double line UV-Vis-NIR spectrophotometer (Perkin Elmer UV/ Vis/NIR lambda 900 , resolution $=1 \mathrm{~nm})$. The sample of BN-SWNTs is placed on one line and a quartz substrate is placed on the reference line. Therefore, optical absorption spectra are background corrected (light source fluctuations, substrate absorption).

In order to have a reference, hexagonal boron nitride has also been studied by means of optical absorption spectroscopy. The optical absorption spectrum of $h$-BN microcrystals [25] sprayed on a quartz substrate is displayed in Fig. 2. It shows a wide line centered at $6.15 \mathrm{eV}$, which is attributed to the saddle point transition $Q_{2}^{-}-Q_{2}^{-}$in the band structure of $h$-BN [14]. The band gap can be estimated to lie between 5.6 and $5.8 \mathrm{eV}$, which is in very good agreement with previous works on $h$-BN optical properties [14,26,27]. The optical density of the sample is relatively large at low energy (below the band gap), indicating a high density of defects in the $h$-BN gap.

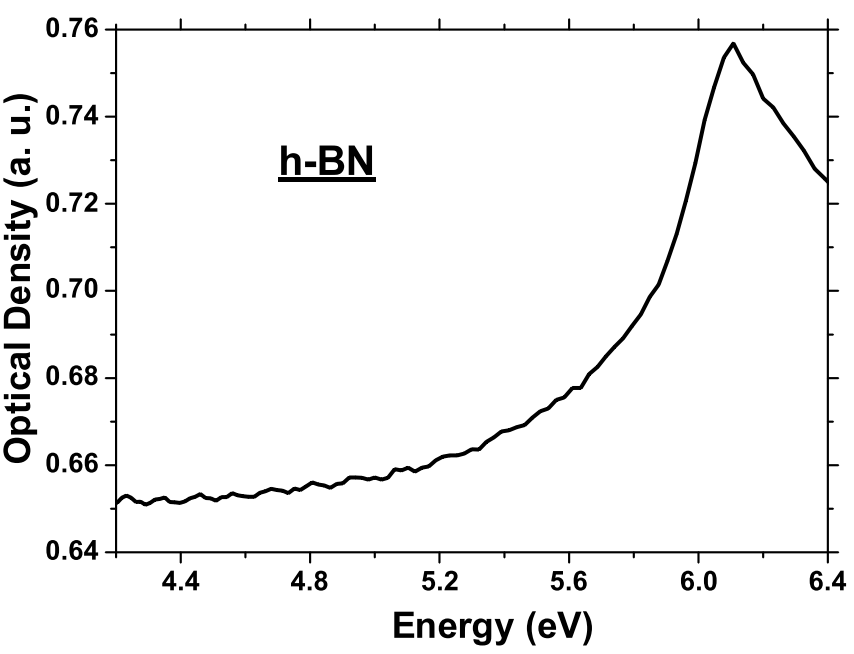

FIG. 2. Optical absorption spectrum of hexagonal boron nitride microcrystals deposited on a quartz substrate.

The optical absorption spectrum of an assembly of BNSWNTs is displayed in Fig. 3. The spectrum has been recorded between 0.7 and $6.5 \mathrm{eV}$. No structure has been observed in the 0.7 to $3 \mathrm{eV}$ region, and the spectrum is then displayed in the 3-6.5 eV range. We observe three absorption lines superimposed on a background. The three lines are centered at 4.45, 5.5, and $6.15 \mathrm{eV}$, respectively (notice that the spectrum is independent of the solvent used in the purification step). The two low energy lines are not present in the $h$-BN optical absorption spectrum. Minor components of the sample as boron oxide, silica particles, or boron particles should not present absorption lines at these energies, to the authors' knowledge. Observations of low energy excitations have also been reported in the case of

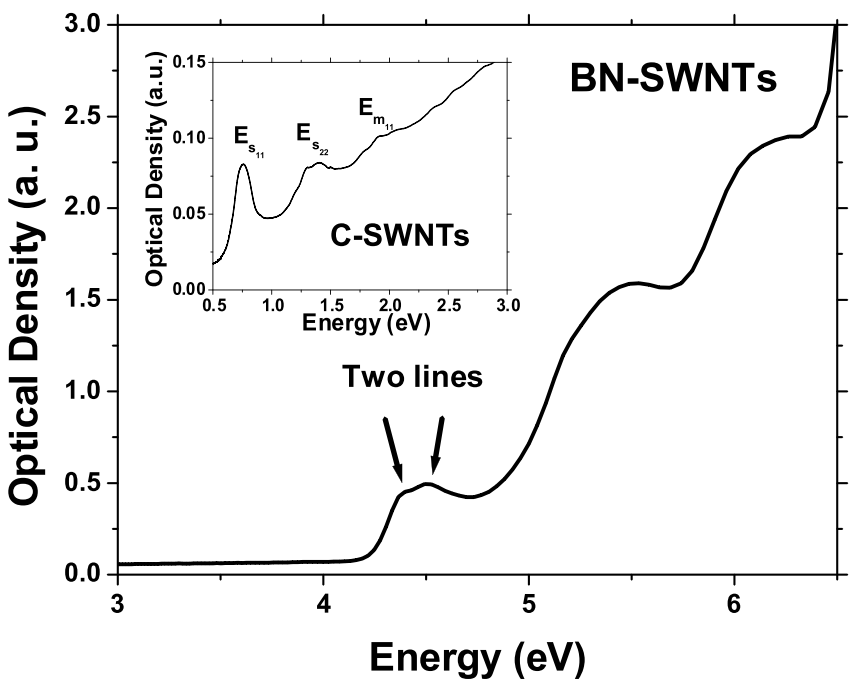

FIG. 3. Optical density of an assembly of single-wall boron nitride nanotubes deposited on a quartz substrate. Inset: Optical absorption spectrum of an assembly of single-wall carbon nanotubes deposited on a glass substrate. 
multiwall $\mathrm{BN}$ nanotubes from the analysis of electron energy loss data [19]. These absorption lines can therefore be understood as the BN-SWNTs contribution. The line width of the lowest energy line is about $340 \mathrm{meV}$. One can observe that this line may be decomposed into two lines centered at 4.4 and $4.5 \mathrm{eV}$, respectively. Finally, the line width of the $5.5 \mathrm{eV}$ line is about $800 \mathrm{meV}$.

For comparison purposes, the inset of Fig. 3 represents the optical absorption spectrum of an assembly of singlewall carbon nanotubes. Three lines $(0.75,1.35$, and $1.95 \mathrm{eV}$ ) superimposed on a strong background are observed. The density of states of carbon nanotubes is composed by pairs of van Hove singularities, due to the quantification involved by the rolling up of the graphene sheet. The lines observed in the absorption spectrum are commonly attributed to the transitions between pairs of such singularities [11].

BN-SWNTs are also one-dimensional materials. The lines at 4.45 and $5.5 \mathrm{eV}$ could therefore be viewed as the result of the quantification due to the rolling up of the $h$-BN sheet. As for carbon nanotubes, the lines could be assigned to the transition between pairs of van Hove singularities in the one-dimensional density of states of BN-SWNTs. In this framework, these results should be compared to those obtain in scanning tunneling spectroscopy (STS) experiments $[17,18]$. However, these experiments are performed on more complex BN nanotubes and the data are highly scattered $(4-8 \mathrm{eV})$. Indeed, the STS results seem to be difficult to analyze because of the insulating feature of these objects [18].

As a matter of fact, the confinement energy due to the rolling up of the $h$-BN sheet cannot explain a fundamental transition energy (gap) of BN-SWNTs $1 \mathrm{eV}$ below the gap of $h$-BN. Furthermore, to the best of our knowledge, all theoretical calculations estimate that BN-SWNT gaps should be around the $h$-BN gap (between 5.5 and $6 \mathrm{eV}$ ) $[20,21]$. These calculations do not take into account electron-hole Coulomb interactions. Therefore, another interpretation of the nature of these lines can be suggested. BN-SWNTs are one-dimensional materials and Coulomb interactions are therefore enhanced. Electron-hole interactions could explain a position of absorption peaks of $\mathrm{BN}-$ SWNTs below the gap of $h$-BN. The line at $4.45 \mathrm{eV}$ could therefore be viewed as an excitonic transition. The exciton binding energy $\left(E_{b}\right)$ would be of the order of $1 \mathrm{eV}$ $(\gg k T)$, which explains that it can be observed at room temperature. This kind of exciton binding energy is in good agreement with calculations performed for BN-SWNTs [28]. Using the electron and hole effective masses in hexagonal boron nitride [29], the reduced mass is about $\mu=0.17 \mathrm{~m}_{0}$ (where $\mathrm{m}_{0}$ is the free electron mass). Then, in a free exciton interpretation, the exciton radius is evaluated to be about $1 \mathrm{~nm}$. The exciton is very localized (not free) and is a so-called Frenkel exciton, as observed in alkali-halide crystals, in rare gas crystals or in molecular crystals [30,31]. The line at $5.5 \mathrm{eV}$ can then be assigned to the transition between the first pair of van Hove singularities of the single particle density of states (i.e., to the single particle band gap, without electron-hole interaction). Assuming the excitonic interpretation of the $4.45 \mathrm{eV}$ line, the energy position of this line cannot be compared with results of STS experiments. In fact, STS experiments probe the one particle density of states, and then do not observe excitons, at least not directly. Finally, the line centered at $6.15 \mathrm{eV}$ is characteristic of the hexagonal boron nitride [14].

As mentioned above, the line centered at $4.45 \mathrm{eV}$ presents two substructures. As in carbon nanotubes, the existence of these substructures, as well as the very large line width, could be attributed to chirality effects. In other words, these features would be the consequence of a distribution of chirality and diameter in the sample. Nevertheless, most theoretical works predict a weak dependence of the electronic properties of BN-SWNTs on diameter and chirality [20,22]. Assume again an exciton interpretation of this line. The exciton of BN-SWNTs is strongly bound and localized. The coupling between excitons and the lattice might be very strong, as it is usually the case for Frenkel excitons [30]. Therefore self-trapping effects might occur and account for the splitting of the exciton line, and also explain the broad line $(340 \mathrm{meV})$ which is observed [32,33], as it is usually the case in molecular materials [31].

In conclusion, we have reported the first experiments on optical transitions in single-wall boron nitride nanotubes. Three optical transitions are observed in the optical absorption spectrum at $4.45,5.5$, and $6.15 \mathrm{eV}$. The two low energy lines are attributed to the BN-SWNTs transitions. The nature of these lines has been discussed and two interpretations are considered. These lines can be viewed as optical transitions between van Hove singularities in the one-dimensional density of states of BN-SWNTs, but an excitonic interpretation of the $4.45 \mathrm{eV}$ line is preferred. A splitting of the low energy line $(4.45 \mathrm{eV})$ is also observed. Assuming the excitonic interpretation of these line, the splitting may put in evidence a strong electron-phonon coupling, in terms of self-trapping effects. These experiments have been performed at room temperature. Some low temperature experiments (optical absorption and photoluminescence spectroscopies) are under progress and would give more information on the nature of the optical absorption lines and on the splitting of the $4.45 \mathrm{eV}$ line. Finally, these first results suggest that BN-SWNTs are promising materials for application in optoelectronics (blue-light source, UV detector, etc.).

The authors are grateful to A. Filoramo and J.P. Bourgoin (LEM, CEA, France) for their help and the use of their chemistry facilities. The authors are also grateful to L. Reining's group (Polytechnique, France), L. Wirtz and A. Rubio (DIPC, Spain), and Ph. Roussignol, 
R. Ferreira, G. Cassabois, and C. Voisin (LPA, ENS, France) for helpful discussions. LEM de l'ONERA is a "Unité mixte de recherche associée au CNRS" (UMR 104). The work was supported by the French Grant "NABOCO" and by the GDR-E NanoE (No. 2756).

*Electronic address: jean-sebastien.lauret@onera.fr [1] S. Iijima et al., Nature (London) 354, 56 (1991).

[2] M. M. Treacy et al., Nature (London) 381, 678 (1996).

[3] A. Bachtold et al., Science 294, 1317 (2001).

[4] M. Freitag et al., Nano Lett. 3, 1067 (2003).

[5] M. J. O'Connel et al., Science 297, 593 (2002).

[6] S. M. Bachilo et al., Science 298, 2361 (2002).

[7] J. Lefebvre et al., Phys. Rev. Lett. 90, 217401 (2003).

[8] J. S. Lauret et al., Phys. Rev. Lett. 90, 057403 (2003).

[9] S. Lebdekin et al., J. Phys. Chem. B 107, 1949 (2003).

[10] J. S. Lauret et al., Physica (Amsterdam) 21E, 1057 (2004).

[11] O. Jost et al., Appl. Phys. Lett. 75, 2217 (1999).

[12] A. Loiseau et al., Phys. Rev. Lett. 76, 4737 (1996).

[13] R. S. Lee et al., Phys. Rev. B 64, 121405 (2001).

[14] A. Zunger et al., Phys. Rev. B 13, 5560 (1976).

[15] B. G. Demczyk et al., Appl. Phys. Lett. 78, 2772 (2001).

[16] M. Radosavljevic et al., Appl. Phys. Lett. 82, 4131 (2003).

[17] R. Czerw et al., Appl. Phys. Lett. 83, 1617 (2003).

[18] M. Ishigami et al., AIP Conf. Proc. 685, 389 (2003).

[19] G. G. Fuentes et al., Phys. Rev. B 67, 035429 (2003).

[20] A. Rubio et al., Phys. Rev. B 49, 5081 (1994).
[21] A. G. Marinopoulos et al., Appl. Phys. A 78, 1157 (2004).

[22] M.F. Ng et al., Phys. Rev. B 69, 115417 (2004).

[23] O. Stephan et al., in Proceedings of the Institute of Physics, Electron Microscopy and Analysis Group, University of Oxford, 2003, edited by S. McVitie and D. McComb (Institute of Physics Publishing, Bristol, 2003), Vol. 179, p. 437.

[24] R. Arenal et al., AIP Conf. Proc. 685, 384 (2003).

[25] $h$-BN microcrystals bought from the Goodfellow Corporation.

[26] M. J. Rand et al., J. Electrochem. Soc. 115, 423 (1968).

[27] A. J. Noreika et al., J. Vac. Sci. Technol. 6, 722 (1969).

[28] It should be emphasized here that a detailed comparison of our results with theoretical ones is still difficult since many corrections to the usual $a b$ initio local-densityapproximation-RPA calculations have to be taken into account: GW corrections to get a reasonable single particle gap, local field effects, and excitonic effects [G. Onida et al., Rev. Mod. Phys. 74, 601 (2002)]. Such calculations also have to be made for $h$-BN as well as for SWNTs isolated or in bundles. They are in progress [L. Wirtz (private communication)].

[29] Y. N. Xu et al., Phys. Rev. B 44, 7787 (1991).

[30] K. S. Song et al., Self Trapped Exciton (Springer-Verlag, Berlin, 1993).

[31] M.I. Ranasinghe et al., J. Am. Chem. Soc. 124, 6520 (2002).

[32] K. Ishida et al., Z. Phys. B 102, 483 (1997).

[33] R. W. Munn et al., Phys. Rev. B 57, 1328 (1998). 